\title{
Análise de trilha usando valores fenotípicos e genotípicos para componentes do rendimento na seleção de famílias de cana-de-açúcar
}

\author{
Path analysis using phenotypic and genotypic values for yield components in the selection of \\ sugarcane families
}

\author{
Deiciana Pagano Espósito ${ }^{\mathrm{I}}$ Luiz Alexandre Peternelli ${ }^{*}$ Thiago Otávio Mendes de Paula ${ }^{\mathrm{II}}$ \\ Márcio Henrique Pereira Barbosa ${ }^{\text {II }}$
}

\section{RESUMO}

\begin{abstract}
O objetivo deste trabalho foi investigar o uso de valores fenotípicos e genotípicos na análise de trilha envolvendo características agronômicas em cana-de-açúcar, nos estágios de cana-planta e cana-soca. Sob esses dois enfoques, foram computados os efeitos diretos e indiretos dos componentes de produção - número de colmos por parcela, diâmetro médio de colmos e altura média de colmos - sobre a produtividade de colmos por hectare. Para garantir uma melhor consistência nos resultados, os dados foram obtidos em dois experimentos, no delineamento em blocos casualizados com cinco repetições. Os experimentos foram alocados no Centro de Experimentação em cana-de-açúcar da UFV, em Oratórios, MG, Brasil. Cada experimento foi constituído de 22 famílias de irmãos completos de cana-de-açúcar. Os coeficientes de determinação foram elevados em todas as análises de trilha, indicando que os componentes avaliados explicam grande parte da variação existente na produção de colmos. Praticamente não houve diferença de interpretações e conclusões entre as análises baseadas nas matrizes de correlações fenotípicas ou genotípicas. Pela análise dos efeitos diretos fenotípicos e genotípicos, o número de colmos foi o caractere que melhor se correlacionou com toneladas de cana por hectare, em ambos os experimentos e estágios. Isso demonstra a possibilidade de obtenção de ganhos significativos por meio da seleção indireta para toneladas de cana por hectare via número de colmos. Houve variação em relação às estimativas de correlação na comparação entre os experimentos, o que provavelmente se deve à origem diferenciada das famílias avaliadas em cada experimento.
\end{abstract}

Palavras-chave: correlação, seleção precoce, seleção indireta.

\section{ABSTRACT}

The aim of this study was to investigate the use of phenotypic and genotypic values in the path analysis involving

\begin{abstract}
agronomic characteristics in sugarcane at the stages of plant cane and ratoon cane. Under these two approaches it was calculated the direct and indirect effects of yield components number of stalks per plot, diameter of stalk and average height of stalk - on sugarcane yield per hectare. To ensure a better consistency of results, data were obtained in two experiments in randomized blocks with five replications. The experiments were carried out at the Center of Sugarcane Experimentation of the UFV, Oratórios, MG, Brazil. Each experiment consisted of 22 full-sib families of sugarcane. The coefficients of determination were high in all path analysis, indicating that the evaluated components explain most of the variation in straw yield. Practically there was no difference in interpretations and conclusions from the analysis based on arrays of genotypic or phenotypic correlations. From the analysis of the direct phenotypic and genotypic effects, number of stalks was the variable that best correlated with sugarcane yield per hectare in both experiments and harvest stages, demonstrating the possibility of obtaining significant gains by means of indirect selection of sugarcane yield per hectare using number of stalks. There was variation in the estimates of correlation in the comparison between experiments, probably due to different origin of the families surveyed in each experiment.
\end{abstract}

Key words: correlation, early selection, indirect selection.

\section{INTRODUÇÃO}

Relações existentes entre os caracteres são, em geral, avaliadas por meio das correlações fenotípicas, genotípicas e ambientais. A correlação fenotípica tem causas genéticas e ambientais, porém, somente as genéticas envolvem uma associação de natureza herdável, podendo, portanto, ser utilizada para

'Departamento de Estatística, Universidade Federal de Viçosa (UFV), 36570-000, Viçosa, MG, Brasil. E-mail: peternelli@ufv.br.

*Autor para correspondência.

IIDepartamento de Fitotecnia, UFV, Viçosa, MG, Brasil. 
orientar programas de melhoramento. Assim, em estudos genéticos, é indispensável distinguir e quantificar o grau de associação genética e ambiental entre os caracteres (CRUZ et al., 2004; MARCHEZAN et al., 2005).

Contudo, podem ocorrer alguns equívocos nas estratégias de seleção das características avaliadas a partir da quantificação da magnitude das correlações entre as variáveis, pois um alto ou baixo coeficiente de correlação entre dois caracteres pode ser resultado do efeito de um terceiro sobre eles, ou de um grupo de caracteres (CRUZ et al., 2004). Assim, para o melhor entendimento dos fenômenos de associação entre as variáveis, o estudo da análise de trilha é indispensável no melhoramento de cana. Este estudo aponta, além dos efeitos diretos, as características mais adequadas para seleção indireta, uma vez que a quantificação da produção é um trabalho demorado devido ao grande número de genótipos avaliados nas etapas iniciais.

KANG et al. (1983) salientam que o rendimento de cana constitui uma característica complexa influenciada por vários caracteres interrelacionados. A interdependência entre os caracteres frequentemente influencia a relação direta com o rendimento e, como resultado, a informação baseada em coeficientes de correlação se torna não confiável. Mas a análise dos coeficientes de trilha permite a decomposição dos coeficientes de correlação em efeitos diretos e indiretos e fornece uma relação mais prática dos caracteres, ajudando na identificação de componentes de grande efeito.

Atualmente, a análise de trilha é amplamente utilizada por melhoristas de plantas, em diversas culturas como o milho (AGRAMA, 2006), o feijão (CABRAL et al., 2011), o arroz (MARCHEZAN et al., 2005), o algodão (HOOGERHEIDE et al., 2007), a soja (BÁRBARO et al., 2006) e o trigo (GONDIM et al., 2008). Estudos sobre relações entre variáveis importantes para a cultura da cana via análise de trilha também são encontrados na literatura (KANG et al., 1983; REDDY \& REDDI, 1986; SUKHCHAIN \& SAINI, 1997; FERREIRA et al., 2007; SILVA et al., 2009). Alguns autores usam correlações fenotípicas enquanto outros afirmam ser melhor as genotípicas. Não há consenso quanto a isso, o que leva à necessidade de novos estudos investigativos.

Considerando a necessidade de aperfeiçoar o processo de identificação de genótipos promissores nos programas de melhoramento genético de cana-deaçúcar, desenvolveu-se o presente trabalho como o objetivo de quantificar, por meio da análise de trilha, com uso dos valores fenotípicos e genotípicos, os efeitos diretos e indiretos dos seus componentes de produção sobre a produtividade de colmos por hectare nos estágios de cana-planta e de cana-soca.

\section{MATERIAL E MÉTODOS}

Os experimentos foram implementados em abril de 2007, no Centro de Pesquisa e Melhoramento da Cana-de-açúcar (CECA), localizado em Oratórios - MG (latitude 205' $\mathrm{S}$, longitude $42^{\circ} 48^{\prime} \mathrm{W}$ e $494 \mathrm{~m}$ de altitude), pertencente à Universidade Federal de Viçosa. Dados de cana-planta e cana-soca da fase inicial de seleção foram obtidos em dois experimentos. Cada experimento constituído de vinte e duas famílias de irmãos completos de cana-de-açúcar. Os cruzamentos foram realizados em 2006, na Estação de Floração e Cruzamentos, localizada na Serra do Ouro, município de Murici - AL, situada à latitude $9^{\circ} 13^{\prime} \mathrm{S}$, longitude $35^{\circ} 50^{\prime} \mathrm{W}$ e a $450-500 \mathrm{~m}$ de altitude. As plântulas obtidas de cada família foram transplantadas conforme metodologia descrita por BARBOSA\& SILVEIRA(2000).

Em cada experimento, foi utilizado o delineamento em blocos casualizados com cinco repetições. A parcela foi composta por dois sulcos espaçados de 1,40m. Cada sulco foi composto por dez plantas equidistantes a $0,5 \mathrm{~m}$. A adubação dos experimentos foi realizada de acordo com aquela recomendada para a cultura (KORNDÖRFER et al., 1999).

As seguintes características foram avaliadas em junho de 2008 (cana-planta) e julho de 2009 (canasoca): i) altura média de colmos $(A C)$ em metros, mensurando-se um colmo (amostrado aleatoriamente) de cada touceira, desde a base do colmo até a primeira folha cuja-seção compreendida entre o limbo foliar e a bainha está visível; ii) diâmetro médio de colmos $(D C)$ em milímetros, com a amostragem feita no quinto entrenó, contado da base do colmo para o ápice, mensurando-se com paquímetro um colmo (amostrado aleatoriamente) de cada touceira; iii) número de colmos por parcela $(N C)$; iv) tonelada de colmos por hectare $\left(T C H_{\text {direito }}\right)$, obtida de forma direta pela expressão $T C H_{\text {direito }}=(m p \times 10) / t p$, em que $m p$ é massa total da parcela (conjunto de colmos sem folhas, cortados rente ao solo) em $\mathrm{kg}$, e tp é o tamanho da parcela $\mathrm{em}^{2}$, sendo, neste trabalho, igual a $14 \mathrm{~m}^{2}$.

Inicialmente, foram obtidas as estimativas das correlações genotípicas (rg) e fenotípicas (rf) entre os caracteres avaliados. Com base nas matrizes de correlação genotípicas e fenotípicas, foi realizado o diagnóstico da multicolinearidade de acordo com o critério "número de condição" (razão entre os autovalores máximos e mínimos das matrizes) indicados por MONTGOMERY \& PECK (1981). Posteriormente, foi realizada a análise de trilha (WRIGHT, 1923; 
FALCONER \& MACKAY, 1996) conforme diagrama causal usual, no qual a produção de colmos é determinada pelos seus componentes $(A C, D C$ e $N C)$, por meio dos efeitos diretos e indiretos de cada um desses componentes.

Todas as análises foram efetuadas com o auxílio do Programa R (R DEVELOPMENT CORE TEAM, 2009).

\section{RESULTADOS E DISCUSSÃO}

Constataram-se diferenças significativas $(\mathrm{P}<0,01)$ entre famílias em relação a todos os caracteres estudados. Observou-se boa precisão experimental em ambos os experimentos, pois a maioria dos caracteres apresentou coeficientes de variação $(\mathrm{CV})$ relativamente baixos (inferiores a 20\%). O diagnóstico de multicolinearidade baseado no número de condição apresentou valores menores que 100 , indicando multicolinearidade classificada como fraca em ambos os casos. Dessa forma, a análise de trilha foi realizada sem necessidade de técnicas estatísticas mais complexas.

A maioria das correlações fenotípicas e genotípicas entre os componentes de produção $(N C$, $D C, A C$ ) com $T C H$ foram altas ou moderadas e positivas, sugerindo, inicialmente, que um aumento em qualquer um desses componentes causaria aumento correspondente em TCH (Tabela1). Resultados semelhantes foram obtidos por FERREIRA et al. (2007) e SILVA et al. (2009). Esses autores também trabalharam com análises de trilha envolvendo as mesmas variáveis e encontraram que esses componentes explicam grande parte da variação existente na $T C H$. Alguma variação de magnitude dos efeitos ocorreu para cada estágio da cana (cana-planta ou cana-soca), sendo que $N C$ foi o componente mais influente em ambos os estágios.

As estimativas dos coeficientes de correlação genotípica e fenotípica, entre os caracteres em estudo, para os estágios de cana-planta e canasoca referentes ao experimento 1 , seguem apresentadas na tabela 1. Embora a correlação genotípica entre $T C H$ e $N C(0,7595)$ seja relativamente alta, esta não foi significativa para o estágio de cana-soca. FERREIRA et al. (2007) também obtiveram correlações genotípicas não significativas entre essas variáveis ao analisarem dados deste mesmo estágio.

Cumpre destacar que a significância da correlação genotípica foi verificada pela expressão $t=r_{g} / \sqrt{\operatorname{vâr}\left(r_{g}\right)}$. Assim, quando a variância da correlação genotípica entre dois caracteres foi alta, o valor encontrado para $t$ foi pequeno, implicando um baixo

Tabela 1 - Estimativas dos coeficientes de correlação fenotípica e genotípica (entre parênteses) entre as variáveis: número de colmos por parcela $(N C)$, diâmetro médio de colmos por parcela $(D C)$, altura média de colmos por parcela $(A C)$ e tonelada de colmos por hectare $(T C H)$, avaliadas em famílias de cana-de-açúcar.

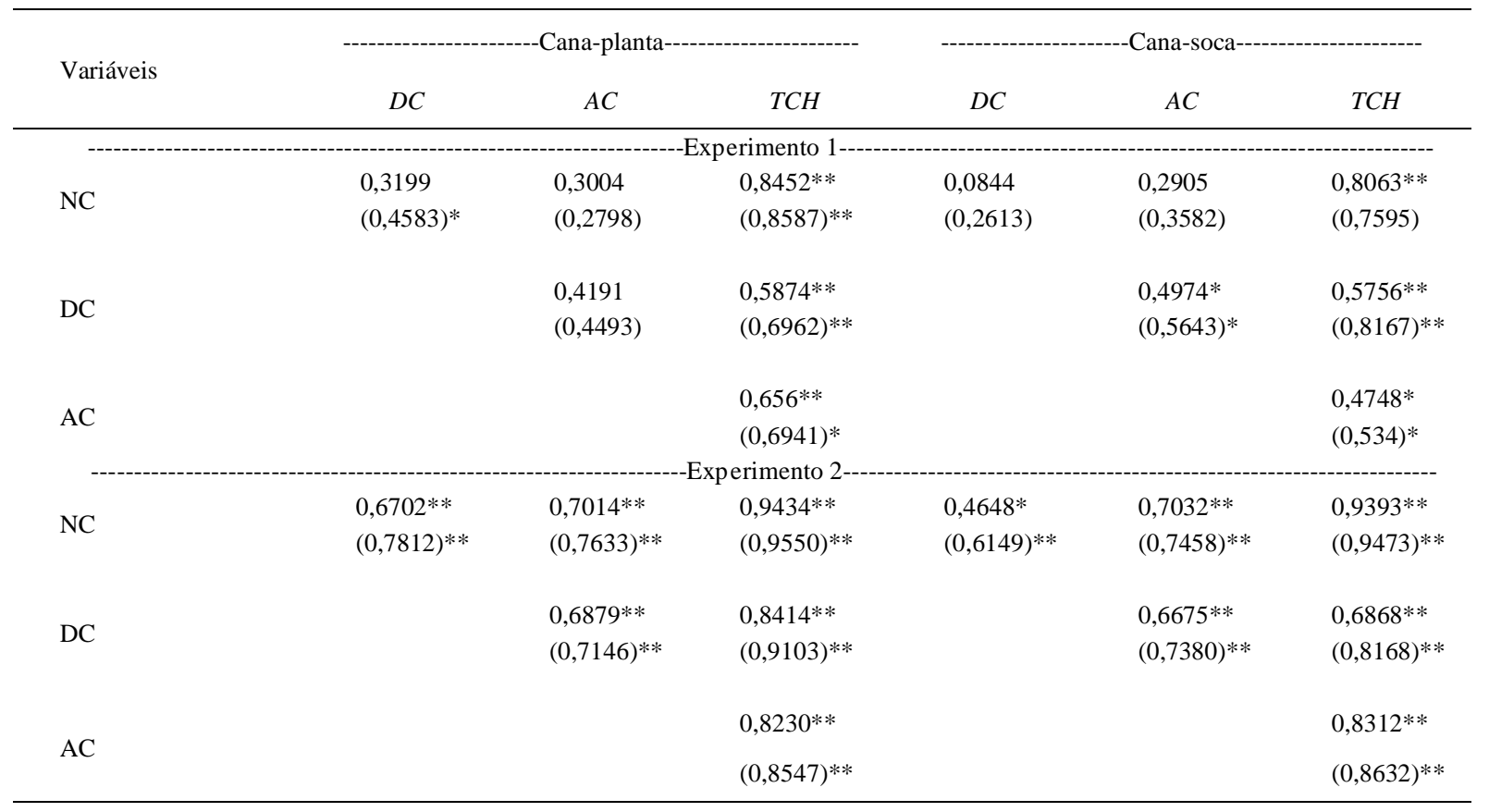

\footnotetext{
${ }^{*, * *}$ Significativo a $1 \%$ e a $5 \%$, respectivamente, pelo teste t.
} 
poder do teste, o que poderia explicar a não significância da correlação genotípica entre $T C H$ e $N C$.

Observou-se alguma concordância de sinais nas correlações fenotípicas e genotípicas para os dois estágios. Quanto às magnitudes, houve ligeira tendência das correlações genotípicas superarem as fenotípicas. Assim, é possível deduzir que os componentes genotípicos têm maior influência na determinação das correlações que os componentes de ambiente sobre o componente fenotípico. No entanto, os valores encontrados para a correlação genotípica podem estar superestimados, uma vez que esta é proveniente de cálculos indiretos a partir de componentes da variância e da covariância. Contudo, KANG et al. (1983) também encontraram correlações fenotípicas e genotípicas com magnitudes próximas, permitindo concluir que a variância e a covariância ambiental foram reduzidas a nível desprezível, isto é, a influência do ambiente sobre estas relações foi pequena.

As maiores estimativas das correlações fenotípicas e genotípicas foram para número de colmos $(N C)$, tanto no estágio de cana-planta quanto no estágio de cana-soca. Considerando apenas os coeficientes de correlação, o componente de produção $N C$ é, portanto, de principal importância na determinação de $T C H$. A estimativa do coeficiente de correlação genotípica entre $D C$ e $T C H$, no estágio de cana-soca $(0,8167)$ no experimento 1 , foi superior ao estágio de cana-planta $(0,6962)$, no entanto, o mesmo caso não ocorreu para os demais caracteres.

Diferentemente do ocorrido no experimento 1, o experimento 2 apresentou todas as estimativas dos coeficientes de correlação fenotípica e genotípica significativas a $1 \%$ pelo teste $\mathrm{t}$ (Tabela 1 ). Contudo, as estimativas das correlações em ambos os experimentos nos levam a conclusões semelhantes. Esses resultados estão concordantes aos encontrados por SILVA et al. (2009), para o estágio de cana-planta.

Desse modo, os coeficientes de correlação das variáveis explicativas $N C, D C$ e $A C$ sobre $T C H$ foram decompostos em efeitos diretos e indiretos, conforme apresentado na tabela 2 . As estimativas dos coeficientes de determinação em cana-planta e canasoca, para ambos os experimentos, explicaram satisfatoriamente as variações em $\mathrm{TCH}$, como indica o alto valor do coeficiente de determinação do modelo e pelo efeito residual baixo. Tal resultado reflete a excelente contribuição das variáveis do modelo para a produção de colmos.

No experimento 2, observou-se que, tanto nas correlações fenotípicas quanto nas genotípicas, os efeitos diretos e indiretos dos caracteres $N C, D C$ e
$A C$ sobre $T C H$ foram todos positivos no estágio de cana-planta. Os diretos foram superiores aos indiretos ao se analisar $N C$ e $A C$. No entanto, para a variável $D C$, os efeitos diretos e indiretos apresentaram, praticamente, as mesmas magnitudes, refletindo os valores de correlações fenotípicas e genotípicas entre as variáveis $D C$ e $N C(0,3199$ e 0,4583 , respectivamente) e $D C$ e $A C(0,4191$ e 0,4493 , respectivamente), fazendo com que houvesse fracionamento por igual do coeficiente de correlação entre os efeitos direto e indireto.

Embora as variáveis $D C$ e $A C$ tenham apresentado estimativas de correlação genotípica e fenotípica moderadas com $\mathrm{TCH}$, os efeitos diretos foram baixos no experimento 1 , sendo o efeito direto da variável $D C(0,2226)$ inferior ao valor de efeito da variável residual $(0,2629)$, o que reduz sua importância em relação às variáveis $N C$ e $A C$. Segundo CRUZ et al. (2004), caracteres com alta correlação favorável, como é o caso da variável $D C$ em cana-planta, com correlação genotípica igual a 0,6962, mas com baixo efeito direto, indicam que a melhor estratégia deverá ser a seleção simultânea de caracteres, com ênfase também nos caracteres cujos efeitos indiretos são significativos.

No estágio de cana-planta, destaca-se $N C$ como a variável que mais contribuiu para explicar $T C H$. Esse resultado está de acordo com os obtidos por SILVA et al. (2009), que obtiveram altos efeitos diretos de $N C$ sobre TCH. Os trabalhos de JAMES (1971) e REDDY \& REDDI (1986) apresentaram contribuição semelhante de $N C$ e $D C$ sobre $T C H$, seguidos de $A C$. SUKHCHAIN et al. (1997) também obtiveram altos efeitos diretos de $N C$ sobre $T C H$, sugerindo a seleção de clones para elevação da produção de colmos com base nesta variável.

Foram encontrados, no estágio de canasoca, efeitos diretos positivos e superiores aos efeitos indiretos entre as variáveis $\mathrm{TCH}$ e $\mathrm{NC}$ e $\mathrm{TCH}$ e $\mathrm{DC}$, tanto para as correlações fenotípicas quanto para as genotípicas no experimento 1 (Tabela 2). Contudo, o efeito direto de $A C$ sobre $T C H$ foi negativo e inferior aos efeitos indiretos, evidenciando a baixa contribuição dessa variável para $T C H$ neste estágio. Possivelmente, a correlação moderada positiva entre $T C H$ e $A C$ está sendo causada pelos efeitos indiretos, via $N C$ e $D C$. De todo modo, o comportamento apresentado pela variável $A C$ em relação à $T C H$ é indicativo da ausência de causa e efeito entre essas variáveis. Segundo CRUZ et al. (2004), nessa situação, o caráter independente nãoé o principal determinante das alterações na variável principal, existindo outros fatores que poderão proporcionar maior impacto em termos de ganhos de seleção. Tais resultados sugerem o desenvolvimento 
Tabela 2 - Análise de trilha fenotípica e genotípica dos componentes de produção número de colmos por parcela $(N C)$, diâmetro médio de colmos por parcela $(D C)$, altura média de colmos por parcela $(A C)$ sobre tonelada de colmos por hectare $(T C H)$.

\begin{tabular}{|c|c|c|c|c|}
\hline & --------------C & ta-------------- & --------------C & a-------------. \\
\hline & Fenotípica & Genotípica & Fenotípica & Genotípica \\
\hline$N C$ & & & & \\
\hline Efeito direto sobre $\mathrm{TCH}$ & 0,6650 & 0,6440 & 0,7636 & 0,6041 \\
\hline Efeito indireto via $D C$ & 0,0712 & 0,0977 & 0,0432 & 0,1839 \\
\hline Efeito indireto via $A C$ & 0,1090 & 0,1170 & $-0,0005$ & $-0,0285$ \\
\hline Total & 0,8452 & 0,8587 & 0,8063 & 0,7595 \\
\hline Efeito direto sobre $\mathrm{TCH}$ & 0,2226 & 0,2132 & 0,5120 & 0,7037 \\
\hline Efeito indireto via $N C$ & 0,2127 & 0,2952 & 0,0644 & 0,1578 \\
\hline Efeito indireto via $A C$ & 0,1521 & 0,1879 & $-0,0008$ & $-0,0449$ \\
\hline Total & 0,5874 & 0,6962 & 0,5756 & 0,8167 \\
\hline Efeito direto sobre $\mathrm{TCH}$ & 0,3630 & 0,4181 & $-0,0017$ & $-0,0795$ \\
\hline Efeito indireto via $N C$ & 0,1998 & 0,1802 & 0,2218 & 0,2164 \\
\hline Efeito indireto via $D C$ & 0,0933 & 0,0958 & 0,2547 & 0,3971 \\
\hline Total & 0,6560 & 0,6941 & 0,4748 & 0,5340 \\
\hline Coef. de determinação $\left(\mathrm{R}^{2}\right)$ & 0,9309 & 0,9916 & 0,9096 & 0,9911 \\
\hline Efeito da variável residual & 0,2629 & 0,0914 & 0,3007 & 0,0945 \\
\hline Efeito direto sobre TCH & 0,6076 & 0,5211 & 0,7041 & 0,6397 \\
\hline Efeito indireto via DC & 0,2051 & 0,2821 & 0,1133 & 0,1870 \\
\hline Efeito indireto via AC & 0,1307 & 0,1519 & 0,1219 & 0,1205 \\
\hline Total & 0,9434 & 0,9550 & 0,9393 & 0,9473 \\
\hline Efeito direto sobre TCH & 0,3060 & 0,3611 & 0,2439 & 0,3041 \\
\hline Efeito indireto via NC & 0,4072 & 0,4071 & 0,3273 & 0,3934 \\
\hline Efeito indireto via AC & 0,1282 & 0,1422 & 0,1157 & 0,1193 \\
\hline Total & 0,8414 & 0,9103 & 0,6868 & 0,8168 \\
\hline Efeito direto sobre $\mathrm{TCH}$ & 0,1863 & 0,1989 & 0,1733 & 0,1616 \\
\hline Efeito indireto via NC & 0,4262 & 0,3977 & 0,4951 & 0,4771 \\
\hline Efeito indireto via DC & 0,2105 & 0,2580 & 0,1628 & 0,2245 \\
\hline Total & 0,8230 & 0,8547 & 0,8312 & 0,8632 \\
\hline Coeficiente de determinação $\left(\mathrm{R}^{2}\right)$ & 0,9841 & 0,9963 & 0,9729 & 0,9840 \\
\hline Efeito da variável residual & 0,1262 & 0,0604 & 0,1647 & 0,0778 \\
\hline
\end{tabular}

de índice de seleção de famílias de cana-de-açúcar utilizando apenas as variáveis $N C$ e $D C$.

No trabalho realizado por KANG et al. (1983), a decomposição dos coeficientes de correlação genotípica mostrou que diâmetro médio de colmos $(D C)$ e número de colmos $(N C)$ produziram igual contribuição para $T C H$, seguido de altura média de colmos $(A C)$. Para as correlações fenotípicas, os três componentes $N C, D C$ e $A C$, apresentaram igual importância para a produção de cana-de-açúcar. De acordo com esses autores, ainda que do ponto de vista prático, os coeficientes de trilha genotípicos deveriam ser mais importantes para decidir sobre um critério de seleção mais eficaz. Contudo, caracteres genotipicamente correlacionados, mas não fenotipicamente correlacionados podem não ser de valor prático na seleção, pois geralmente esta é baseada no fenótipo (RESENDE, 2002). Por isso, os dois tipos de correlação foram considerados neste estudo para facilitar a decisão sobre a eficiência de um critério de seleção indireta. 
No estágio de cana-soca no experimento 2, os efeitos diretos dos componentes de produção $D C$ e $A C$ foram baixos, à semelhança do estágio de canaplanta, evidenciando a baixa contribuição dessas variáveis para $T C H$. O componente de produção $N C$ apresentou a maior estimativa de efeito direto sobre $T C H$, para a correlação fenotípica, bem como para a correlação genotípica, à semelhança dos resultados obtidos anteriormente. Esse resultado indica que em ambos os experimentos, constituídos de diferentes famílias e estágios, esse componente teve maior contribuição para a produção de cana-de-açúcar. Verifica-se também que houve aumento desse efeito direto em relação ao estágio de cana-planta, o que pode ser justificado pela redução dos efeitos indiretos de $N C$ via $D C$ e $A C$ (Tabela 2).

O rendimento de cana constitui uma característica complexa influenciada por vários caracteres inter-relacionados, sendo a análise dos coeficientes de trilha uma importante estatística na ajuda quanto à identificação dos componentes de grande efeito. Assim, a variável $N C$, de fácil mensuração, foi o componente que apresentou maior contribuição para a produção de cana-de-açúcar, com possibilidade de obtenção de ganhos significativos por meio da seleção indireta para $T C H$ via $N C$, em ambos os experimentos e estágios.

Destaca-se ainda que a variação na magnitude das estimativas de correlação apresentada entre os experimentos se deve, provavelmente, à origem diferenciada das famílias avaliadas. Sendo assim, evidencia a necessidade de avaliação de maior número de experimentos.

\section{CONCLUSÃO}

As informações e conclusões obtidas pelo uso da matriz de correlação fenotípica ou genotípica foram semelhantes. A variável $N C$ foi o componente que apresentou maior contribuição para a produção de canade-açúcar, com possibilidade de obtenção de ganhos significativos por meio da seleção indireta para $T C H$ via $N C$ ou por meio de índice de seleção via $N C$ e $D C$.

\section{AGRADECIMENTOS}

\footnotetext{
À Fundação de Amparo à Pesquisa de Minas Gerais (FAPEMIG), e ao Conselho Nacional de Pesquisa (CNPq), pelo apoio financeiro.
}

\section{REFERÊNCIAS}

AGRAMA, H.A.S. Sequential path analysis of grain yield and its components in maize. Plant Breeding, v.115, p.343-346, 2006.
BÁRBARO, I.M. et al. Path analysis and expected response in indirect selection for grain yield in soybean. Crop Breeding and Applied Biotechnology, v.6, p.151-159, 2006. Disponível em: <http://www.sbmp.org.br/cbab/siscbab/uploads/bd6ba09c512e-6051.pdf $>$. Acesso em: 10 mar. 2011.

BARBOSA, M.H.P.; SILVEIRA, L.C.I. Metodologias de seleção, progressos e mudanças no programa de melhoramento genético da cana-de-açúcar da Universidade Federal de Viçosa. STAB, Açúcar, Álcool e Subprodutos, v.18, p.30-32, 2000.

CABRAL, P.D.S. et al. Análise de trilha do rendimento de grãos de feijoeiro (Phaseolus vulgaris L.) e seus componentes. Revista Ciência Agronômica, v.42, p.132-138, 2011. Disponível em: 〈http://www.scielo.br/pdf/rca/v42n1/v42n1a17.pdf >. Acesso em: 10 mar. 2011. doi: 10.1590/S180666902011000100017

CRUZ, C.D. et al. Modelos biométricos aplicados ao melhoramento genético. Viçosa: UFV, 2004. 390p.

FALCONER, D.S.; MACKAY, T.F.C. Introduction to quantitative genetics. Edinburgh: Longman Group Limited, 1996. 464p.

FERREIRA, F.M. et al. Relações fenotípicas e genotípicas entre componentes de produção em cana-de-açúcar. Bragantia, v.66, p.605-610, 2007. Disponível em: <http://www.scielo.br/ pdf/brag/v66n4/10.pdf >. Acesso em: 10 mar. 2011. doi: 10.1590/S0006-87052007000400010.

GONDIM, T.C.O. et al. Análise de trilha para componentes do rendimento e caracteres agronômicos de trigo sob desfolha. Pesquisa Agropecuária Brasileira, v.43, p.487-493, 2008. Disponível em: <http://www.scielo.br/pdf/pab/v43n4/ a07v43n4.pdf>. Acesso em: 10 maio, 2011. doi:10.1590/ S0100-204X2008000400006.

HOOGERHEIDE, E.S.S. et al. Correlações e análise de trilha de caracteres tecnológicos e a produtividade de fibra de algodão. Pesquisa Agropecuária Brasileira, v.42, p.1401-1405, 2007. Disponível em: <http://www.scielo.br/pdf/pab/v42n10/ a05v4210.pdf $>$. Acesso em: 10 mar. 2011. doi: 10.1590/ S0100-204X2007001000004.

JAMES, N.I. Yield components in random and selected sugarcane populations. Crop Science, v.11, p.906-908, 1971.

KANG, M.S. et al. Genetic and phenotypic path analysis and heritability in sugarcane. Crop Science, v. 23, p.643-647, 1983.

KORNDÖRFER, G.H. et al. Sugestões de adubação para canade-açúcar. In: RIBEIRO, A.C. et al. Recomendações para o uso de corretivos e fertilizantes em Minas Gerais. 5 aproximação. Viçosa, MG: Comissão de Fertilidade do Solo do Estado de Minas Gerais, 1999. p.285-288.

MARCHEZAN, E. et al. Análise de coeficiente de trilha para os componentes de produção em arroz. Ciência Rural, v.35, p.1027-1033, 2005. Disponível em: <http://www.scielo.br/pdf/ cr/v35n5/a07v35n5.pdf >. Acesso em: 10 mar. 2011. doi: 10.1590/S0103-84782005000500007.

MONTGOMERY, D.C.; PECK, E.A. Introduction to linear regression analysis. New York: John Wiley \& Sons, 1981. 504p. 
R DEVELOPMENT CORE TEAM R: a language and environment for statistical computing. Austria: R Foundation for Statistical Computing, Vienna, 2009. Disponível em: <http:/ /www.R-project.org>. Acesso em: 25 nov. 2009.

REDDY, C.R.; REDDI, M.V. Degree of genetic determination, correlation and genotypic and phenotypic path analysis of cane and sugar yield in sugarcane. Indian Journal of Genetics and Plant Breeding, v.46, p.550-557, 1986.

RESENDE, M.D.V. Genética biométrica e estatística no melhoramento de plantas perenes. Brasília: Embrapa Informação Tecnológica, 2002. 975p.
SILVA, F.L. et al. Análise de trilha para os componentes de produção de cana-de-açúcar via Blup. Revista Ceres, v.56, p.308-314, 2009. Disponível em: <http://www.ceres.ufv.br/ceres/ revistas/V56N003P52809.pdf>. Acesso em: 10 mar. 2011.

SUKHCHAIN, S.D.; SAINI, G.S. Inter-relationships among cane yield and commercial cane sugar and their component traits in autumn plant crop of sugarcane. Euphytica, v.95, p.109-114, 1997.

WRIGHT, S. Theory of path coefficients. Genetics, v.8, p.239-285, 1923 . 